\title{
Influence of the Granularity on the Transversal Resistance in $\mathrm{Y}_{0.55} \mathrm{Pr}_{0.45} \mathrm{Ba}_{2} \mathrm{Cu}_{3} \mathrm{O}_{7-\delta}$ Superconductor
}

\author{
M.S. DA LUZ ${ }^{a, b, *}$, C.A.M. DOS SANTOS ${ }^{b}$ AND A.J.S. MACHADO ${ }^{b}$ \\ ${ }^{a}$ Instituto de Ciências Tecnológicas e Exatas, Universidade Federal do Triângulo Mineiro, Uberaba, Brazil \\ ${ }^{b}$ Departamento de Engenharia de Materiais, Escola de Engenharia de Lorena-USP, Brazil
}

(Received October 18, 2010; in final form August 29, 2012)

\begin{abstract}
This work reports longitudinal $\left(R_{X X}\right)$ and transverse $\left(R_{X Y}\right)$ resistance as a function of temperature measured at low magnetic fields for $\mathrm{Y}_{0.55} \mathrm{Pr}_{0.45} \mathrm{Ba}_{2} \mathrm{Cu}_{3} \mathrm{O}_{7-\delta}$ polycrystalline sample. It is observed non-zero transverse resistance at zero applied magnetic field below superconducting transition. The comparison between $R_{X X}(T)$ and $R_{X Y}(T)$ curves demonstrate unambiguously that the transverse resistance is related to the double resistive superconducting transition in granular superconductors. $I-V$ curves indicate that the transport properties of the investigated sample are in agreement with the predictions of the two-fluid model.
\end{abstract}

DOI: 10.12693/APhysPolA.124.702

PACS: 74.25.-q, 74.25.Fy, 74.81.Bd

\section{Introduction}

The transverse resistance in the superconducting state is one of the major tools for studying vortex dynamics and has attracted great attention during the past decades [1-15]. According to the classical model, transverse resistance should have the same sign as the normal state due to normal currents flowing through the vortex core $[16,17]$. However, one of the most interesting behaviors observed in high critical temperature $\left(T_{\mathrm{C}}\right)$ and in some conventional superconductors is the sign reversal of the Hall voltage which changes from positive at normal state to negative near the critical temperature [1-5]. Additionally, a second sign reversal of the Hall resistance was observed in several samples of high- $T_{\mathrm{C}}$ superconductors (HTSC) [5-8]. This double sign reversal the Hall resistivity is positive in the normal state, changes to negative near $T_{\mathrm{C}}$, and becomes positive again at low temperatures. In order to explain this behavior, Göb et al. [2] considered the Hall conductivity $\left(\sigma_{X Y}\right)$ to be composed of three components related to the quasiparticle or vortex-core contributions which is associated with the normal state excitations $\left(\sigma_{X Y}^{\mathrm{N}}\right)$, superconducting dissipation resulting from hydrodynamic vortex effects or superconducting fluctuations $\left(\sigma_{X Y}^{\mathrm{S}}\right)$, and pinning dependence $\left(\sigma_{X Y}^{\mathrm{P}}\right)$. The Hall conductivity is calculated by using those components in the following way:

$\sigma_{X Y}=\sigma_{X Y}^{\mathrm{N}}+\sigma_{X Y}^{\mathrm{S}}+\sigma_{X Y}^{\mathrm{P}}$.

More recently, great attention has been given to the observation of a transverse voltage at zero external magnetic field in HTSC near the superconducting transition [9-14]. Glazman [10] proposed a model in which the magnetic field produced by applied current can create vortex and antivortex in the edges of the sample. Those vor-

*corresponding author; e-mail: daluz.mario@icte.uftm.edu.br tices move into opposite directions under the influence of the Lorentz force and can annihilate each other when the attractive interaction between them overcomes the Lorentz force. In other words, it means that the paths of the vortex and antivortex can be distorted and a transverse voltage should appear according to the Josephson relation [13]. Furthermore, Janeček and Vašek [11, 12] introduced the idea of guiding force which should influence the motion of the vortex and antivortex.

On the other hand, effects of weak coupling between grains or superconducting clusters have been extensively studied in polycrystalline samples [18-23]. The main observed effect is the broadening of the superconducting transition which has been separated in two distinct regimes. Below the onset of the critical temperature, $T_{\mathrm{C} i}$, the reduction of the resistance is related to the formation of isolated superconducting clusters. Furthermore decrease of the temperature leads to the connection of the superconducting clusters mediated by the Josephson coupling at a temperature labeled $T_{\mathrm{C} j}$. Such a regime is non-ohmic and as the temperature decreases an infinite network of connected superconducting clusters leads the sample to a zero-resistance superconducting state. This effect has been often related to branching points in longitudinal resistance curves measured under different applied currents $[18,21]$. It is also determined by clockwise hysteresis loops in magnetoresistance measurements [19-21]. Previous measurements performed in polycrystalline samples of the $\mathrm{Y}_{1-x} \mathrm{Pr}_{x} \mathrm{Ba}_{2} \mathrm{Cu}_{3} \mathrm{O}_{7-\delta}$ $(\mathrm{Y} 123+\mathrm{Pr}), \mathrm{Bi}_{2} \mathrm{Sr}_{2} \mathrm{Ca}_{1-x} \mathrm{Pr}_{x} \mathrm{Cu}_{2} \mathrm{O}_{8-\delta}(\mathrm{Bi} 2212+\mathrm{Pr})$, and $\mathrm{Sm}_{2-x} \mathrm{Ce}_{x} \mathrm{CuO}_{4-\delta}$ systems at low magnetic fields have shown that the dissipation is clearly dominated by weak coupling mechanisms [21-23]. Based upon these measurements we have recently suggested that the non-ohmic regime at temperatures lower than $T_{\mathrm{C} j}$ is described by two-fluid model for granular superconductors [23]. In order to study more detail about this mechanism, in this work the effect of the granularity on the Hall conductivity of the $\mathrm{Y}_{0.55} \mathrm{Pr}_{0.45} \mathrm{Ba}_{2} \mathrm{Cu}_{3} \mathrm{O}_{7-\delta}$ polycrystalline sample is 
reported. We shall prove that the features observed in the transversal resistance are clearly connected to the wide transition observed in granular superconducting samples.

\section{Experimental procedure}

Polycrystalline sample of $\mathrm{Y}_{0.55} \mathrm{Pr}_{0.45} \mathrm{Ba}_{2} \mathrm{Cu}_{3} \mathrm{O}_{7-\delta}$ composition was prepared by solid-state reaction technique using high purity $\mathrm{Y}_{2} \mathrm{O}_{3}, \mathrm{Ba}_{2} \mathrm{CO}_{3}, \mathrm{Pr}_{6} \mathrm{O}_{11}$, and $\mathrm{CuO}$ powders. The powders were calcined at $800^{\circ} \mathrm{C}$ in air for $24 \mathrm{~h}$. The calcination was repeated twice with intermediate grinding. The compounds were then pressed into pellets and sintered in air at $900{ }^{\circ} \mathrm{C}$ for $48 \mathrm{~h}$. The sintered samples were characterized by powder X-ray diffraction. The homogeneity of the grains has been observed by scanning electron microscopy. The sample was cut into a square shape with $7.2 \mathrm{~mm}$ in width and $0.7 \mathrm{~mm}$ thickness. The electrical contacts were placed on the corners of the sample as shown schematically in the inset of Fig. 1.

To study the Hall effect we have measured longitudinal and transverse voltages using the Van der Pauw technique at low magnetic field $(H<100$ Oe) alternating the electrical current in order to eliminate thermo-power effects produced in the voltage contacts. Details about the electrical resistance measurements are given in Refs. [13, 14].

\section{Results and discussion}

Figure 1a displays the longitudinal resistance $\left(R_{X X}\right)$ as a function of the temperature measured at zero applied magnetic field for different applied current. From these measurements one can see clearly the appearance of the double resistive superconducting transition. Two distinct transition temperatures, $T_{\mathrm{C} i}$ and $T_{\mathrm{C} j}$, can be seen which is described by the weak coupling behavior such as discussed by some authors [18-23]. Between $T_{\mathrm{C} i}$ and $T_{\mathrm{C} j}$ the drop in the electrical resistance is related to the formation of isolated superconducting clusters which reduce the equivalent resistance of the samples decreasing temperature. This current dependence has been suggested to be related to the Josephson coupling between superconducting clusters which leads the sample to a zero-resistance superconducting state at low applied current with decreasing temperature (see $R_{X X}(T)$ curve for $I=1 \mathrm{~mA}$ ).

In Fig. 1b we have shown the typical magnetic field dependence of the longitudinal resistance for granular superconducting samples. A broadening of superconducting transition is observed in the $R_{X X}(T)$ measurements. With the change of the value of magnetic field, $T_{\mathrm{C} i}$ is almost constant. Above $T_{\mathrm{C} i}$ the resistance changes significantly with change in magnetic field. This behavior is associated with weak links between the superconducting clusters [23].

In order to study the dissipation due to vortex motion, we have performed longitudinal magnetoresistance, $R(H)$, measurements. It is important to note that these $R(H)$ curves were taken at $4.2 \mathrm{~K}$ with the sample within the He bath in order to avoid temperature fluctuations.

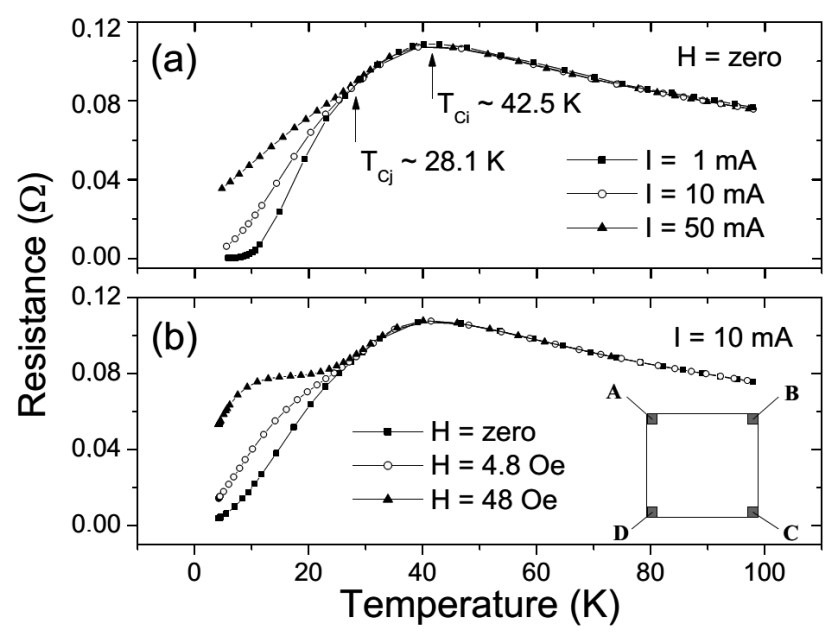

Fig. 1. (a) Longitudinal electrical resistance as a function of temperature, $R_{X X}(T)$, measured at different applied currents for the sample $\mathrm{Y}_{0.55} \mathrm{Pr}_{0.45} \mathrm{Ba}_{2} \mathrm{Cu}_{3} \mathrm{O}_{7-\delta}$. In (b) $R_{X X}(T)$ measured at different applied magnetic fields are shown. Inset displays a schematic view of the electrical contacts used during the measurements.

Figure 2 presents the behavior of the magnetoresistance for three applied currents. A careful inspection of these curves reveals a common feature, clockwise hysteresis loops in the range of applied magnetic field studied. Such a clockwise hysteresis is accompanied by the presence of a crossover field $\left(H^{*} \approx 2.5 \mathrm{Oe}\right)$. The magnitude of the electrical resistance in the decreasing branch of the curve is always lower than that noticed in the increasing curve, for $H>H^{*}$. The opposite behavior occurs for $H<H^{*}$, the magnitude of $R(H)$ is always lower in the increasing branch of the curve. The interpretation of this behavior involves the motion of the Josephson vortices in the intergranular region which is in agreement with the observations of clockwise hysteresis loops reported by other authors [19-22].

From the results of Fig. 2, starting with the sample in the mixed state (no zero resistance), increasing $H$ results in a substantial increase of the magnitude of $R(H)$ and the electrical resistivity reaches a saturation value close to 20 Oe. Such saturation value corresponds to the electrical resistance of a material with isolated superconducting islands embedded in an insulating matrix. Decreasing $H$ from the saturation value, the Josephson vortices can move relatively freely. Thus, as $H$ decreases and reaches values far away from the maximum, a partial fraction of the Josephson vortices move out of the unpinned regions first while others stay trapped within the sample. Following this explanation the resistance observed in the decreasing branch of $R(H)$ is lower than taken during increasing $H$. These results demonstrated unambiguously that dissipation at low temperatures $\left(T<T_{\mathrm{C} j}\right)$ is due to weak coupling between superconducting cluster such as pointed out in previous reports [19-22]. 


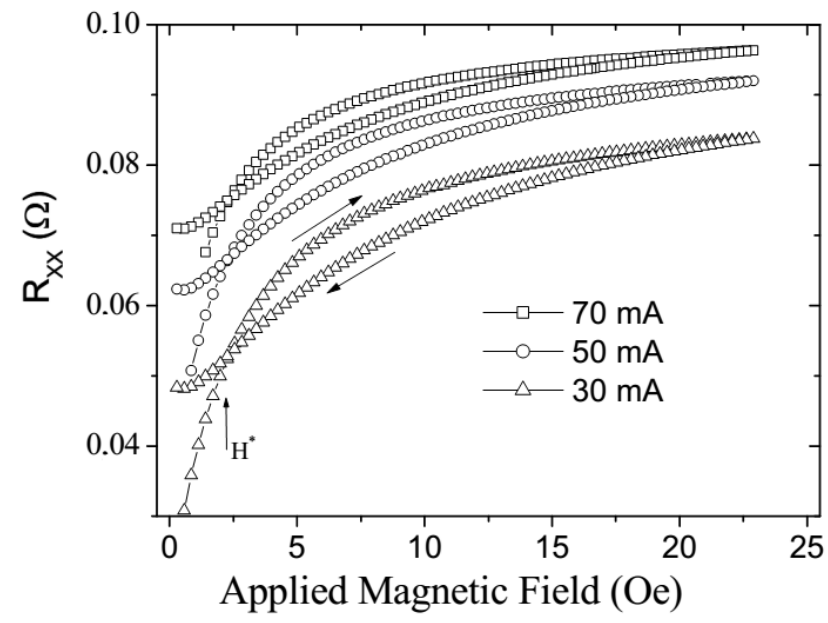

Fig. 2. Magnetoresistance, $R(H)$, hysteresis loops measured at $4.2 \mathrm{~K}$ for three different applied currents.

Figure 3 displays longitudinal and transverse resistance as a function of the temperature measured with field of 4.8 Oe applied normal to the sample, for three different applied currents. Details about the electrical resistance measurements are given in Refs. [13, 14]. In the longitudinal component one can see the broadening of the superconducting transition increasing applied current and the appearance of the two distinct transition temperatures, $T_{\mathrm{C} i}$ and $T_{\mathrm{C} j}$, which is described by the weak coupling behavior such as discussed by some authors [18-23]. On the other hand, in the transverse resistance is possible to observe several important features. The transverse resistance shows strong temperature dependence above $T_{\mathrm{C}}$ but less in the normal state. In the normal state the signal is positive but as the temperature is lowered to the superconducting state, $R_{X Y}$ becomes negative. Toward lower temperature a second sign reversal back to positive is also observed. The sign reversal of transverse voltage has also been observed by other authors [1-5].

The most important feature in Fig. 3 is the relationship between the sign reverse in transverse resistance curves and the transition temperatures $T_{\mathrm{C} i}$ and $T_{\mathrm{C} j}$, observed in the longitudinal resistance measurements. The change in $R_{X Y}$ from positive (in the normal state) to negative is related to the intergranular transition temperature $T_{\mathrm{C} i}$. On the other hand, the second sign reversal back to positive is clear connected to the intergranular transition temperature $T_{\mathrm{C} j}$. The $R_{X Y}$ broadening, below $T_{\mathrm{C} j}$, is clear related to intergranular or weak coupling effects. Based upon the results, discussed above, weak coupling or granular effects should be taken into account in the transverse resistance measurements.

In the inset of Fig. 3a measurements taken at zero applied magnetic field are shown. One can see that the non-zero transverse voltage appears in the superconducting transition. The appearance of a transverse resistance at zero applied magnetic field was attributed to attraction

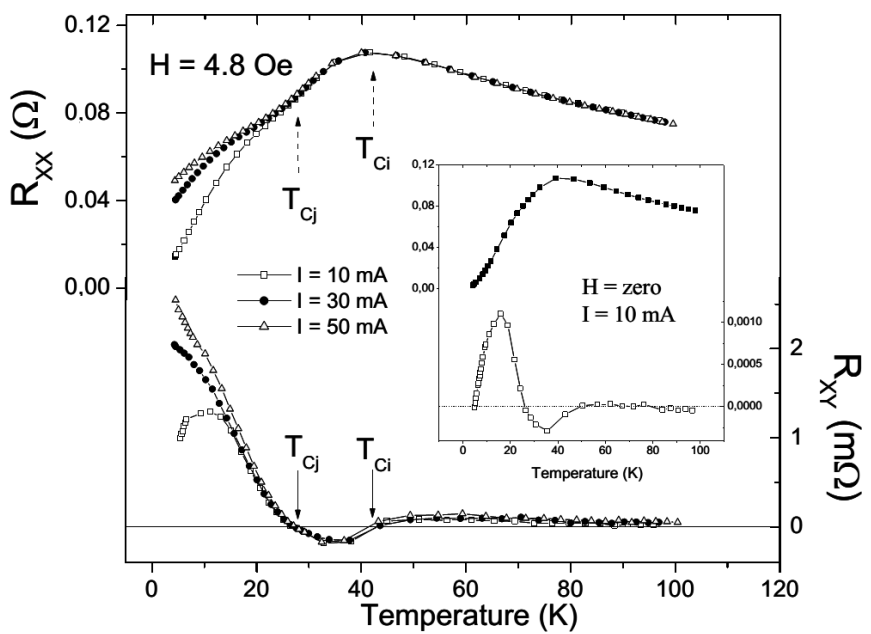

Fig. 3. Longitudinal (upper curves) and transverse (lower curves) resistance components measured for different applied currents at $H=4.8$ Oe. Inset displays the same components at zero applied magnetic field for $I=10 \mathrm{~mA}$.

among vortex and antivortex, generated at two opposite edges of a sample by the self field of the applied current $[9,10]$. Attractive vortex-antivortex interaction modifies the vortex trajectory by providing velocity components to the current, thus generating a local electric field transverse to the current direction. Polarity of this local field depends on the trajectory of individual vortices. Similar results have been reported by several authors [11-15].

To gain further information concerning transport properties of this granular superconductor sample we have performed $I-V$ curves. Figure 4 displays the transversal $V_{X Y}(I)$ and longitudinal $V_{X X}(I)$ components in applied magnetic fields up to 95 Oe. The longitudinal components display a typical $I-V$ behavior of superconducting granular materials which reveal the double resistive superconducting transition, as those reported by Santos et al. [23]. Most importantly the data displayed in $V_{X X}(I)$ show that, in the high current limit, the slopes of the $I-V$ characteristics are magnetic field independent (see inset). At high excitation currents, the $\mathrm{d} V / \mathrm{d} I$ curves approach a constant value, a feature which is magnetic field independent. Inset highlights the $V_{X X}(I)$ at high applied current limit. These results indicate that the transport properties of the investigated sample are in agreement with the predictions of the two-fluid model [23]. In the two-fluid model it is supposed that both applied magnetic field and electrical current are related to the weak coupling effects and they are not high enough to reach the limits expected to break the Cooper pairs or induce the Abrikosov vortices inside superconducting samples. There are two carriers type, normal electrons and Cooper pairs, coexisting in equilibrium at temperatures below $T_{\mathrm{C}}$. This implies that normal electrons contribute also to the transversal voltage in granular superconductors. Back to Fig. 4, the $V_{X Y}(I)$ 


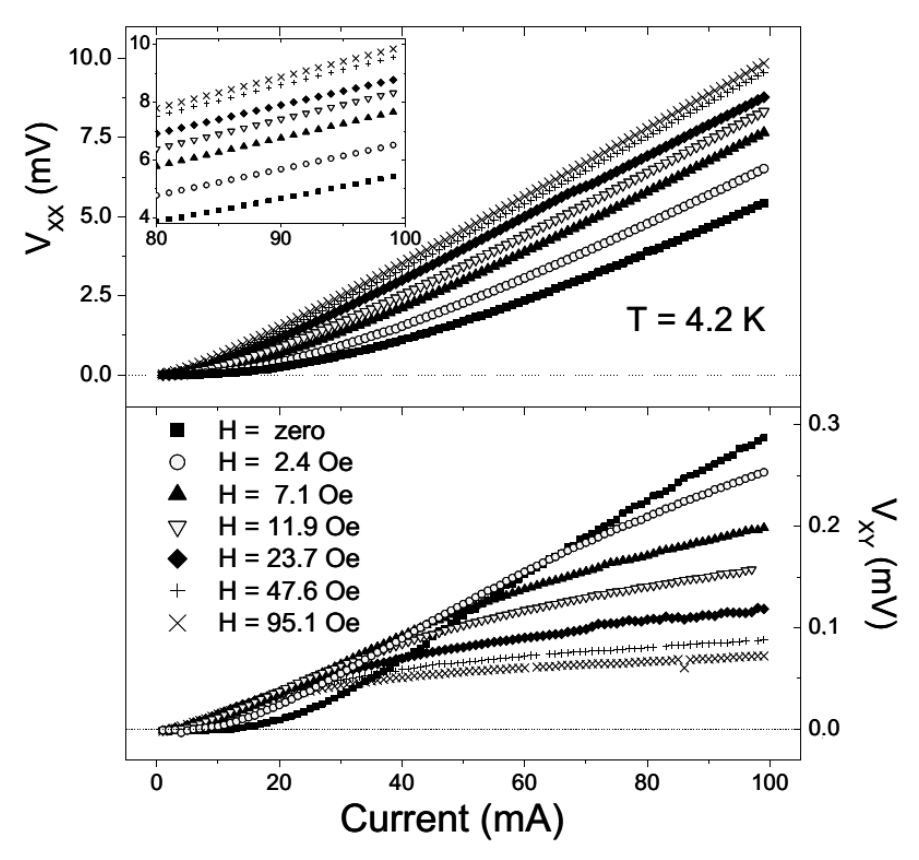

Fig. 4. $I-V$ curves for the longitudinal $V_{X X}(I)$ and transverse $V_{X Y}(I)$ components in the mixed state $(T=$ $4.2 \mathrm{~K}$ ) measured under different applied magnetic fields. Inset highlights the $V_{X X}(I)$ at high applied current limit.

components are strongly field dependent. Furthermore, as the current is increased $V_{X Y}$ values decrease which suggests that the normal electrons and the intergranular vortex and antivortex have opposite contribution to the transverse resistance.

\section{Conclusion}

This work reports longitudinal and transverse resistance as a function of the temperature measured in $\mathrm{Y}_{0.55} \mathrm{Pr}_{0.45} \mathrm{Ba}_{2} \mathrm{Cu}_{3} \mathrm{O}_{7-\delta}$ polycrystalline sample at low magnetic field. Transverse resistance measurements displayed double sign reversal which is clearly related to weak coupling effects. We noticed a relationship between the sign reverse in transverse resistance curves and the transition temperatures $T_{\mathrm{C} i}$ and $T_{\mathrm{C} j}$, observed in the longitudinal resistance measurements. Based upon the results, discussed above, weak coupling or granular effects should be taken into account in the transverse resistance measurements.

\section{Acknowledgments}

This work was supported by FAPESP (00/03610-4 and 97/11113-6), FAPEMIG, and CNPq.

\section{References}

[1] W. Lang, W. Göb, J.D. Pedarnig, R. Rössler, D. Bäuerle, Physica C 364-365, 518 (2001).

[2] W. Göb, W. Liebich, W. Lang, I. Puica, R. Sobolewski, R. Rössler, J.D. Pedarnig, D. Bäuerle, Phys. Rev. B 62, 9780 (2000).

[3] A.V. Saimolov, Phys. Rev. B 49, 1246 (1994).

[4] A.W. Smith, T.W. Clinton, C.C. Tsuei, C.J. Lobb, Phys. Rev. B 49, 12927 (1994).

[5] M.N. Kunchur, D.K. Christen, C.E. Klabunde, J.M. Phillips, Phys. Rev. Lett. 72, 2259 (1994).

[6] W.N. Kang, S.H. Yun, J.Z. Wu, D.H. Kim, Phys. Rev. B 55, 621 (1997).

[7] K. Nakao, K. Hayashi, T. Utagawa, Y. Enomoto, N. Koshizuka, Phys. Rev. B 57, 8662 (1998).

[8] H. Khosroabadi, V. Daadmehr, M. Akhavan, Physica C 384, 169 (2003).

[9] T.L. Francavilla, E.J. Cukauskas, L.H. Allen, P.R. Broussard, IEEE Appl. Sup. 5, 1717 (1995).

[10] L.I. Glazman, Sov. Low. Temp. Phys. 12, 389 (1986).

[11] I. Janeček, P. Vašek, Physica C 390, 330 (2003).

[12] P. Vašek, H. Shimakage, Z. Wang, Physica C 411, 164 (2004).

[13] M.S. da Luz, F.J.H. de Carvalho Jr., C.A.M. dos Santos, C.Y. Shigue, A.J.S. Machado, R. Ricardo da Silva, Physica C 419, 71 (2005).

[14] M.S. da Luz, F.J.H. de Carvalho Jr., C.A.M. dos Santos, C.Y. Shigue, A.J.S. Machado, Physica C 469, 60 (2009).

[15] A. Segal, M. Karpovski, A. Gerber, Phys. Rev. B 83, 094531 (2011).

[16] J. Bardeen, M.J. Stephen, Phys. Rev. 140, A1197 (1965).

[17] P. Nozières, W.F. Vinen, Philos. Mag. 14, 667 (1966).

[18] A. Gerber, T. Grenet, M. Cyrot, J. Beille, Phys. Rev. Lett. 65, 3201 (1990).

[19] Y. Kopelevich, V.V. Lemanov, V.V. Makarov, Sov. Phys. Solid State 32, 2095 (1990).

[20] L. Ji, M.S. Rzchowski, N. Anand, M. Tinkham, Phys. Rev. B 47, 470 (1993).

[21] C.A.M. dos Santos, M.S. da Luz, B. Ferreira, A.J.S. Machado, Physica C 391, 345 (2003).

[22] M.J.R. Sandim, R.F. Jardim, Physica C 328, 246 (1999).

[23] C.A.M. dos Santos, C.J.V. Oliveira, M.S. da Luz, A.D. Bortolozo, M.J.R. Sandim, A.J.S. Machado, Phys. Rev. B 74, 184526 (2006). 